\title{
EVOLUTION IN MULTIDIMENSIONAL SPACES
}

\author{
Anatoliy Pogorui \\ Communicated by A. Turbin
}

\begin{abstract}
In this paper, we study evolutions in multidimensional spaces. By reducing multidimensional cases to one-dimensional, the properties of random evolutions in some semi-Markov media are studied.
\end{abstract}

Keywords. Random evolution, Brownian motion, Semi-Markov process..

2010 Mathematics Subject Classification. Primary 60J25; Secondary 60J65.

\section{Introduction}

The Goldstein-Kac telegraph process models a random motion of particles with constant velocity and it is usually proposed as an alternative to diffusion models. This basic telegrapher process was introduced by S.Goldstein and M.Kac in the mid-50s of the 20th century. The process has been extended in many manners since then [1]- [7] and references therein.

A great deal of research devoted to generalization of the telegraph process to higher-dimensional spaces $\mathbb{R}^{n}, n \geq 2$ [4], [6], [7] and other. The main difficulty in multidimensional cases is a continuum of possible directions. This difficulty can be remedied by discretization of possible directions and there are many papers devoted to random motions with a finite number of directions of particle motion [6]- [8] and the bibliography therein.

It should be noted that all papers on random motions in multidimensional spaces are devoted to analysis of models in which motions are driven by a homogeneous Poisson process, so their processes are Markovian [4], [6]- [8] and references therein.

In this paper, it is considered a non-Markovian generalization of the telegrapher's random process to higher dimensions where motion is driven by some semiMarkov processes. We investigate random motions with uniformly distributed on the unit sphere directions in higher dimension by reducing it to the one-dimensional random motion. In so doing, we reduce the study of random motion in multidimensional spaces to the study of well known renewal-reward processes. 


\section{Random motion in multidimensional spaces}

Suppose $\theta_{k}>0, k=1,2, \ldots$ are i.i.d random variables with a cumulative distribution function (cdf) $G(t)$ and there exists the probability density function (pdf) $g(t)=\frac{d G(t)}{d t}$. Let us put $\tau_{m}=\Sigma_{k=1}^{m} \theta_{k}, m=1,2, \ldots, \tau_{0}=0$ and consider the process $\xi(t)=\max \left\{m \geq 0: \tau_{m} \leq t\right\}$.

We assume that the particle moves in $n$-dimensional space $\mathbb{R}^{n}$ in the following manner: Starting at the origin of coordinates $(0,0, \ldots, 0) \in \mathbb{R}^{n}$ the particle continues its motion with constant velocity which norm is $v>0$ and direction is a unit vector $\vec{\eta}_{0}^{(n)}$ with uniform distribution on the unit sphere

$$
\Omega^{n}=\left\{\left(x_{1}, x_{2}, \ldots, x_{n}\right): x_{1}^{2}+x_{2}^{2}+\ldots+x_{n}^{2}=1\right\} .
$$

Then at each instant $\tau_{m}, m \geq 1$ the particle takes on the new direction with uniform distribution on $\Omega^{n}$.

Denote by $\vec{x}^{(n)}(t)$ the particle's position at time $t>0$. Then $\vec{x}^{(n)}(t)$ in anytime $\mathrm{t}$ can be expressed as follows

$$
\vec{x}^{(n)}(t)=v \sum_{i=1}^{\xi(t)} \vec{\eta}_{i}^{(n)} \theta_{i}+v \vec{\eta}_{\xi(t)}^{(n)}\left(t-\tau_{\xi(t)}\right)
$$

Eq. (2.1) determines the random evolution of the particle in the semi-Markov media $\xi(t)$.

Our purpose is to investigate the distribution of the particle position $\vec{x}^{(n)}(t)$ at time $t>0$.

Let us denote by $x^{(n)}(t)$ the length of the projection of $\vec{x}^{(n)}(t)$ on a fixed line, say the $x_{1}$-axis. The probabilistic properties of a random vector are completely determined by those of its projection on $x_{1}$-axis, and using the latter it is frequently possible to avoid analysis in $n$ dimensions. It is important to know the relationship between the distribution function of $\vec{x}^{(n)}(t)$ and the cdf of $x^{(n)}(t)$.

It is shown in [9] that the probability density function $f_{\eta_{i}^{(n)}}(x)$ of the length $\eta_{i}^{(n)}$ of the projection of $\vec{\eta}_{i}^{(n)}$ on a fixed line is as follows

$$
f_{\eta_{i}^{(n)}}(x)= \begin{cases}\frac{\Gamma\left(\frac{n}{2}\right)}{\sqrt{\pi} \Gamma\left(\frac{n-1}{2}\right)}\left(1-x^{2}\right)^{\frac{n-3}{2}}, & |x| \leq 1 ; \\ 0, & |x|>1 .\end{cases}
$$

Therefore, the distribution function $F(t)$ of $\eta_{i}^{(n)} \theta_{i}$ is of the following form 


$$
F(t)= \begin{cases}\frac{1}{2}+\frac{\Gamma\left(\frac{n}{2}\right)}{\sqrt{\pi} \Gamma\left(\frac{n-1}{2}\right)} \int_{0}^{1} G\left(\frac{t}{x}\right)\left(1-x^{2}\right)^{\frac{n-3}{2}} d x, \quad t \geq 0 . \\ \frac{1}{2}-\frac{\Gamma\left(\frac{n}{2}\right)}{\sqrt{\pi} \Gamma\left(\frac{n-1}{2}\right)} \int_{0}^{1} G\left(-\frac{t}{x}\right)\left(1-x^{2}\right)^{\frac{n-3}{2}} d x, \quad t<0 ;\end{cases}
$$

Let us denote by $\widehat{x}^{(n)}(t)=x^{(n)}\left(\tau_{\xi(t)}\right)$ the particle position at the instant $\xi(t)$.

It is easy to see that $\widehat{x}^{(n)}(t)$ is a renewal-reward process of the following form

$$
\widehat{x}^{(n)}(t)=v \sum_{i=1}^{\xi(t)} \eta_{i}^{(n)} \theta_{i}
$$

Therefore, the study of the multidimensional random motion at renewal epochs may be reduce to the investigation of renewal-reward processes [10]. These processes have been studied for a long time and there exists a great variety of works devoted to renewal-reward processes, say papers on large deviations [11] and references therein. Consequently, these results can be applied for the study of random motions.

\section{Random motion in three-dimensional space}

The cdf $F(t)$ of $\eta_{i}^{(3)} \theta_{i}, i \geq 1$. Since $\eta_{i}^{(3)}$ is uniformly distributed on $[-1,1]$ it is easy to see that $F(t)=P\left(\eta_{i}^{(3)} \theta_{i} \leq t\right)$ has the following form

$$
F(t)= \begin{cases}\frac{1}{2}+\frac{1}{2} \int_{0}^{1} G\left(\frac{t}{x}\right) d x, & t \geq 0 \\ \frac{1}{2}-\frac{1}{2} \int_{0}^{1} G\left(-\frac{t}{x}\right) d x, & t<0\end{cases}
$$

For the pdf $f(t)=\frac{d}{d t} F(t)$ we have $f(t)=\frac{1}{2} \int_{t}^{+\infty} \frac{g(x)}{x} d x$, whence for $t \geq 0$

$$
t \frac{d}{d t} f(t)=-\frac{1}{2} g(t)
$$

and for $t<0$

$$
t \frac{d}{d t} f(t)=-\frac{1}{2} g(-t)
$$

Suppose that $\theta_{k}, k \geq 1$ are Erlang-2 distributed with pdf $g(t)=\lambda^{2} t e^{-\lambda t}$, $\lambda>0, t>0$. Substituting it into Eqs.(3.1),(3.2), we get

$$
f(t)=\frac{1}{2} \lambda e^{-\lambda|t|} .
$$


Therefore, the cdf $F_{\widehat{x}}(y)$ of $\widehat{x}^{(3)}(t)=x^{(3)}\left(\tau_{\xi(t)}\right)$ is of the following form

$$
F_{\widehat{x}}(y)=P\left(v \sum_{i=0}^{\xi(t)} \eta_{i} \theta_{i} \leq y\right)=\sum_{k=0}^{\infty} P\left(v \sum_{i=0}^{k} \eta_{i} \theta_{i} \leq y\right) P(\xi(t)=k)
$$

Let us denote by $\varphi(z, t)=E e^{-i z \widehat{x}^{(3)}(t)}=\int_{-\infty}^{\infty} e^{-i z y} d F_{\widehat{x}}(y)$ the characteristic function of $\widehat{x}^{(3)}(t)$.

It is easy to see that

$$
\begin{aligned}
P(\xi(t)=k) & =P\left(\tau_{k} \leq t\right)-P\left(\tau_{k+1} \leq t\right)=G^{*(k)}(t)-G^{*(k+1)}(t) \\
& =e^{-\lambda t}\left(\frac{(\lambda t)^{2 k}}{2 k !}+\frac{(\lambda t)^{2 k+1}}{(2 k+1) !}\right),
\end{aligned}
$$

where $G(t)=1-e^{-\lambda t}-\lambda t e^{-\lambda t}$ and $G^{*(k)}(t)$ is the $k$-fold convolution of $G(t)$.

Whence, in view of Eq.(3.3), we have

$$
\begin{aligned}
\varphi(z, t) & =\sum_{k=0}^{\infty}\left(\frac{\lambda^{2}}{\lambda^{2}+v^{2} z^{2}}\right)^{k} P(\xi(t)=k) \\
& =e^{-\lambda t} \sum_{k=0}^{\infty}\left(\frac{\lambda^{2}}{\lambda^{2}+v^{2} z^{2}}\right)^{k}\left(\frac{(\lambda t)^{2 k}}{2 k !}+\frac{(\lambda t)^{2 k+1}}{(2 k+1) !}\right) \\
& =\frac{e^{-\lambda t}}{2}\left(e^{\frac{\lambda^{2}}{\sqrt{\lambda^{2}+v^{2} z^{2}}} t}+e^{\frac{-\lambda^{2}}{\sqrt{\lambda^{2}+v^{2} z^{2}}} t}\right) \\
& +\left(\lambda^{2}+v^{2} z^{2}\right) \frac{e^{-\lambda t}}{2 \lambda^{2}}\left(e^{\frac{\lambda^{2}}{\sqrt{\lambda^{2}+v^{2} z^{2}}} t}-e^{\frac{-\lambda^{2}}{\sqrt{\lambda^{2}+v^{2} z^{2}}} t}\right) .
\end{aligned}
$$

Suppose $\lambda \rightarrow+\infty$ and $v \rightarrow+\infty$ such that $\frac{v^{2}}{\lambda} \rightarrow \sigma^{2}>0$ then it follows from Eq.(3.4) that

$$
\varphi(z, t) \rightarrow e^{-\frac{1}{2} \sigma^{2} t z^{2}} .
$$

Therefore, $\widehat{x}^{(3)}(t)$ converges weakly to the Wiener process and consequently $\vec{x}^{(3)}(t)$ converges weakly to the Wiener process in $\mathbb{R}^{3}$.

Now suppose that $\theta_{k}, k \geq 1$ have the Maxwell pdf $g(t)=\sqrt{\frac{2}{\pi}} t^{2} e^{-\frac{t^{2}}{2}}$, then it follows from Eqs.(3.1),(3.2) that the random variable $\varsigma_{i}=\eta^{(3)} \theta_{i}$ has the normal distribution as follows

$$
f(t)=\frac{1}{\sqrt{2 \pi}} e^{-\frac{t^{2}}{2}} .
$$


For this case the characteristic function $\varphi(\lambda, t)=E e^{-\lambda i \widehat{x}^{(3)}(t)}$ has the following form

$$
\varphi(\lambda, t)=\sum_{k=0}^{\infty} e^{-\frac{k \lambda^{2} v^{2}}{2}}\left(G^{*(k)}(t)-G^{*(k+1)}(t)\right) .
$$

Then passing to Fourier's transform of $\varphi(\lambda, t)$ w.r.t. $t$, we have

$$
\varphi(\lambda, s)=\int_{-\infty}^{\infty} e^{-i s t} \varphi(\lambda, t) d t=\sum_{k=0}^{\infty} e^{-\frac{k \lambda^{2} v^{2}}{2}} \varphi^{k}(s)(1-\varphi(s))
$$

where $\varphi(s)$ is the characteristic function of the Maxwell distribution

$$
\varphi(s)=\frac{2}{\sqrt{\pi}} \sum_{l=0}^{\infty} \frac{(i \sqrt{2} s)^{l}}{l !} \Gamma\left(\frac{3+l}{2}\right)
$$

\section{Random motion in five-dimensional space}

It follows from the lemma that the pdf $f_{\eta_{i}^{(5)}}(x)$ of $\eta_{i}^{(5)}$ has the following form

$$
f_{\eta_{i}^{(5)}}(x)= \begin{cases}\frac{3}{4}\left(1-x^{2}\right), & x \in[-1,1] \\ 0, & x \bar{\epsilon}[-1,1] .\end{cases}
$$

The cdf of $\eta_{i}^{(5)} \theta_{i}$ is as follows

$$
F(t)=P\left(\eta_{i}^{(5)} \theta_{i} \leq t\right)= \begin{cases}\frac{1}{2}+\frac{3}{4} \int_{0}^{1} G\left(\frac{t}{x}\right)\left(1-x^{2}\right) d x, & t \geq 0 \\ \frac{1}{2}-\frac{3}{4} \int_{0}^{1} G\left(-\frac{t}{x}\right)\left(1-x^{2}\right) d x, & t<0 .\end{cases}
$$

Suppose that there exists the pdf $g(t)=\frac{d G(t)}{d t}$, then for $t \geq 0$ we have

$$
f(t)=\frac{d F(t)}{d t}=\frac{3}{4} \int_{0}^{1} g\left(\frac{t}{x}\right) \frac{1}{x}\left(1-x^{2}\right) d x,
$$

and for $t<0$

$$
f(t)=\frac{d F(t)}{d t}=\frac{3}{4} \int_{0}^{1} g\left(-\frac{t}{x}\right) \frac{1}{x}\left(1-x^{2}\right) d x .
$$


Assume that $\theta_{k}, k=0,1,2, \ldots$ are Erlang distributed with the pdf $g(t)=$ $\frac{1}{6} \lambda^{4} t^{3} e^{-\lambda t}, \lambda>0$. It is easily verified that the pdf of the random variable $\eta_{i}^{(5)} \theta_{i}$ has the form

$$
f(t)=\frac{1}{4}\left(\lambda+\lambda^{2}|t|\right) e^{-\lambda|t|} .
$$

It may be proved that

$$
f(t)=\frac{1}{4}\left(\lambda+\lambda^{2}|t|\right) e^{-\lambda|t|}=\frac{\lambda^{2}}{4} \int_{-\infty}^{\infty} e^{-\lambda|t-u|} e^{-\lambda|u|} d u
$$

Hence, $\eta_{i}^{(5)} \theta_{i}$ can be presented as follows $\eta_{i}^{(5)} \theta_{i}=\xi_{i}+\xi_{i}^{\prime}$, where $\xi_{i}, \xi_{i}^{\prime}$ are i.i.d. random variables with the Laplace distribution $f_{\xi}(t)=\frac{1}{2} \lambda e^{-\lambda|t|}$.

Therefore, the cdf $F_{\widehat{x}}(y)$ of $\widehat{x}^{(5)}(t)=x^{(5)}\left(\tau_{\xi(t)}\right)$ is of the form

$$
F_{\widehat{x}}(y)=P\left(v \sum_{i=0}^{\xi(t)} \eta_{i}^{(5)} \theta_{i} \leq y\right)=\sum_{k=0}^{\infty} P\left(v \sum_{i=0}^{k} \eta_{i}^{(5)} \theta_{i} \leq y\right) P(\xi(t)=k) .
$$

It is easy to see that

$$
P(\xi(t)=k)=e^{-\lambda t}\left(\frac{(\lambda t)^{4 k}}{4 k !}+\frac{(\lambda t)^{4 k+1}}{(4 k+1) !}+\frac{(\lambda t)^{4 k+2}}{(4 k+2) !}+\frac{(\lambda t)^{4 k+3}}{(4 k+3) !}\right)
$$

Let us denote by $\varphi(z, t)=E e^{-i z \widehat{x}^{(5)}(t)}=\int_{-\infty}^{\infty} e^{-i z y} d F_{\widehat{x}}(y)$ the characteristic function of $\widehat{x}^{(5)}(t)$.

Whence, taking into account Eq.(4.1),(4.2), we have 


$$
\begin{aligned}
& \varphi(z, t)=\sum_{k=0}^{\infty}\left(\frac{\lambda^{2}}{\lambda^{2}+v^{2} z^{2}}\right)^{2 k} P(\xi(t)=k) \\
& =e^{-\lambda t} \sum_{k=0}^{\infty}\left(\frac{(\lambda t)^{4 k}}{4 k !}+\frac{(\lambda t)^{4 k+1}}{(4 k+1) !}+\frac{(\lambda t)^{4 k+2}}{(4 k+2) !}+\frac{(\lambda t)^{4 k+3}}{(4 k+3) !}\right) \\
& =\frac{e^{-\lambda t}}{4}\left\{e^{\frac{\lambda^{2}}{\sqrt{\lambda^{2}+v^{2} z^{2}}} t}+e^{\frac{-\lambda^{2}}{\sqrt{\lambda^{2}+v^{2} z^{2}}} t}+e^{\frac{i \lambda^{2}}{\sqrt{\lambda^{2}+v^{2} z^{2}}} t}+e^{\frac{-i \lambda^{2}}{\sqrt{\lambda^{2}+v^{2} z^{2}}} t}\right. \\
& +\frac{\lambda^{2}+v^{2} z^{2}}{\lambda^{2}}\left(e^{\frac{\lambda^{2}}{\sqrt{\lambda^{2}+v^{2} z^{2}}} t}-e^{\frac{-\lambda^{2}}{\sqrt{\lambda^{2}+v^{2} z^{2}}} t}-i\left(e^{\frac{i \lambda^{2}}{\sqrt{\lambda^{2}+v^{2} z^{2}}} t}-e^{\frac{-i \lambda^{2}}{\sqrt{\lambda^{2}+v^{2} z^{2}}} t}\right)\right) \\
& +\left(\frac{\lambda^{2}+v^{2} z^{2}}{\lambda^{2}}\right)^{2}\left(e^{\frac{\lambda^{2}}{\sqrt{\lambda^{2}+v^{2} z^{2}}} t}+e^{\frac{-\lambda^{2}}{\sqrt{\lambda^{2}+v^{2} z^{2}}} t}-\left(e^{\frac{i \lambda^{2}}{\sqrt{\lambda^{2}+v^{2} z^{2}}} t}+e^{\frac{-i \lambda^{2}}{\sqrt{\lambda^{2}+v^{2} z^{2}}} t}\right)\right) \\
& \left.+\left(\frac{\lambda^{2}+v^{2} z^{2}}{\lambda^{2}}\right)^{3}\left(e^{\frac{\lambda^{2}}{\sqrt{\lambda^{2}+v^{2} z^{2}}} t}+e^{\frac{-\lambda^{2}}{\sqrt{\lambda^{2}+v^{2} z^{2}}} t}+i\left(e^{\frac{i \lambda^{2}}{\sqrt{\lambda^{2}+v^{2} z^{2}}} t}+e^{\frac{-i \lambda^{2}}{\sqrt{\lambda^{2}+v^{2} z^{2}}} t}\right)\right)\right\}
\end{aligned}
$$

Similarly to Eq.(3.4),(3.5), it follows from Eq.(4.3) that if $\lambda \rightarrow+\infty$ and $v \rightarrow$ $+\infty$ such that $\frac{v^{2}}{\lambda} \rightarrow \sigma^{2}>0$ then $\widehat{x}^{(5)}(t)$ converges weekly to the Wiener process and consequently $\vec{x}^{(5)}(t)$ converges weakly to the Wiener process in $\mathbb{R}^{5}$.

Analogously, we can study random motions in the Euclidean space $\mathbb{R}^{n}$ for all $n \geq 2$.

\section{Bibliography}

[1] T. Lange and I. E. Shparlinski, Distribution of some sequences of points on elliptic curves, Journal of Mathematical Cryptology 1 (2007), 1-11.

[2] J. D. King, Finite presentability of Lie algebras and pro-p groups, Ph.D. thesis, University of Cambridge, 1995.

[1] M. Kac, A stochastic model related to the telegrapher's equation. Rocky Mountain J. Math. 4 (1974), 497-509.

[2] V. S. Korolyuk and A. F. Turbin, Mathematical Foundations of the State Lumping of Large Systems, Kluwer Academic Publishers, 1994.

[2] V. S. Korolyuk and V. V. Korolyuk, Stochastic Models of Systems, Kluwer Academic Publishers, 1998.

[3] M. A. Pinsky Lectures on Random Evolution, World Scientific Publishing, New Jersey, 1991. 
[4] A. D. Kolesnik and A. F. Turbin, The equation of symmetric Markovian random evolution in a plane, Stoc. Proc. Appl. 75 (1998), 67-87.

[5] A. F. Turbin, Mathematical model of one-dimensional Brownian motion as alternative to mathematical model of Einstein, Wiener, Levy. Fractal Analysis and Related Fields 2 (1998), 47-60. (in Russian)

[6] A. Lachal, Cyclic random motions in $\mathbb{R}^{d}$-space with $n$ directions. ESAIM : Probab.Stat. 10, 277-316 Rocky Mountain J. Math. 10 (2006), 277-316.

[7] E. Orsinger and A. De Gregorio, Random flights in higher spaces, J.Theor.Probab. 20 (2007), 769-806.

[8] A. Di Crescenzo and B. Martinucci A damped telegraph random process with logistic stationary distribution, J.Appl.Probab. 47, Number 1 (2010), 84-96.

[9] A. A. Pogorui, Fading evolution in multidimensional spaces, Ukr. Math. J. 62, Number 11 (2010), 1828-1834.

[10] D. Cox and W. Smith, Renewal Theory, Wiley, New York, 1962.

[11] A. A. Borovkov and K. A. Borovkov, Large deviation probabilities for generalized renewal processes with regularly varying jump emphSiberian Advances in Mathematics 16 (2006), 1-65.

[3] C. Bonnafé and M. J. Dyer, Semidirect product decomposition of Coxeter groups, preprint, 2008. http://arxiv .org/abs/0805.4100.

[4] M. Ciet, T. Lange, F. Sica and J.-J. Quisquater, Improved algorithms for efficient arithmetic on elliptic curves using fast endomorphisms, in: Advances in Cryptology - Eurocrypt 2003, Lecture Notes in Computer Science 2656, pp. 388-400, Springer, Berlin, 2003.

[5] J. D. King, Finite presentability of Lie algebras and pro-p groups, Ph.D. thesis, University of Cambridge, 1995.

[6] T. Lange and I. E. Shparlinski, Distribution of some sequences of points on elliptic curves, Journal of Mathematical Cryptology 1 (2007), 1-11.

[7] S. Schmitt and H. G. Zimmer, Elliptic Curves - A Computational Approach, de Gruyter Studies in Mathematics 31, Walter de Gruyter, Berlin New York, 2003.

Received 15 November, 2010; revised 23 February, 2012.

\section{Author information}

Anatoliy Pogorui, Shelushkova Str. 10, 222, 10008 Zhytomyr, Ukraine.

E-mail: pogor@zu .edu.ua 